\title{
Analyzing the compressive behavior of porous Ti6Al4V by X-ray microtomography
}

\author{
Iván Farias ${ }^{a}$, Luís Olmos $^{b *}$, Omar Jimenez ${ }^{a}$, Héctor Javier Vergara-Hernández $^{c}$, Didier Bouvard ${ }^{d}$, \\ Pedro Gárnicac ${ }^{c}$ Martín Flores ${ }^{a}$ \\ ${ }^{a}$ Departamento de Ingeniería de Proyectos, Universidad de Guadalajara, 45100, Zapopan, \\ Jalisco, México. \\ ${ }^{b}$ Instituto de Investigaciones en Ciencias de la Tierra - INICIT, Universidad Michoacana de San Nicolás \\ de Hidalgo, 58060, Morelia, Michoacán, México. \\ 'Instituto Tecnológico de Morelia, División de Estudios de Posgrado e Investigación, Tecnológico \\ Nacional de México - TecNM, 58120, Morelia, Michoacán, México. \\ ${ }^{d}$ Science et Ingénierie des Matériaux et Procédés - SIMaP, Université Grenoble Alpes, 38000 \\ Grenoble, France.
}

Received: May 22, 2017; Revised: July 04, 2017; Accepted: July 21, 2017

\begin{abstract}
Samples with $40 \%$ vol. of pores and a pore size distribution between 100 and $500 \mu \mathrm{m}$ were produced by powder metallurgy from Ti6Al4V alloy powders. Sintering was performed at $1300{ }^{\circ} \mathrm{C}$ during one hour in an inert Argon atmosphere in a vertical dilatometer. The compressive strength and the porosity of these samples was investigated before and after compression tests through X-ray microtomography. The values of the elastic modulus $(8 \mathrm{GPa})$ and yield strength $(80 \mathrm{MPa})$ are within the range of those used in bone implants. Porosity leads to greater deformation whereas fracture of compacts occurs perpendicularly to the applied load. It was determined that the origin of the failure is generated by rupture of interparticle necks and, large pores enhance the propagation of cracks.
\end{abstract}

Keywords: Porosity, microtomography, compression, 3D images, Ti6Al4V.

\section{Introduction}

Nowadays, different materials such as stainless steel, Co and Ti based alloys have emerged to produce the next generation of biomedical implants ${ }^{1-6}$. One of the major problems to overcome is to reduce or to accommodate the mechanical properties, as they are most of the time higher than those found in human bones. In order to solve this problem, porous materials have become an interesting alternative ${ }^{7}$. However, when ceramic materials are used for these applications there are some difficulties due to their low toughness compared to that of bone. Contrary to this, metal alloys provide better elasticity when used as implants, nevertheless, their corrosion resistance is relatively poor due to body fluids attack, as well as their bioactivity. In a previous study, Oshida ${ }^{8}$ compiled the biocompatibility range for common biomaterials, where a decreasing order in biocompatibility was found as follows: niobium, tantalum, titanium, zirconium, aluminum, 316L and molybdenum. Among those materials, the Ti6A14V alloy is preferred because its overall combination of properties is good, for instance, its mechanical properties can be modified by introducing artificial pores to obtain similar values to those of the human bone ${ }^{9,10}$. Ryan et al. ${ }^{11}$ reviewed the different techniques for manufacturing porous materials using in biomedical applications, the most common being the inclusion of salt particles which are usually removed before or during the sintering process. Regularly, the induced porosities are large because they can stimulate osseointegration. According to Camron et al. ${ }^{12}$, pore size between 100 and $400 \mu \mathrm{m}$ is necessary to promote osseointegration, although, Bobyn et al. ${ }^{13}$ observed that there is a good bone growth in pores smaller than $50 \mu \mathrm{m}$. Another important feature is the interconnectivity of the porosity in order to allow the passage of fluids and substances that nourish the bones. This has received less importance as it is difficult to quantify by conventional characterization techniques. However, the use of computerized microtomography (CMT) allows the analysis of samples in 3D without any damage to them, also, it has been widely used in the characterization of materials manufactured by powder metallurgy mainly, because of the good contrast obtained between the solid and porous phases ${ }^{14,15}$. 
The present work focuses on the fabrication and characterization of highly porous Ti6Al4V alloy materials. In where, quantitatively data of the porosity were assessed through CMT analysis such as; volume fraction, size distribution and interconnectivity before and after compression tests, thus; the effect of the porosity on the compression properties is evaluated.

\section{Materials and Methods}

\subsection{Sample preparation}

Powders of the Ti6Al4V alloy with spherical particles with a size distribution between 45 and $100 \mu \mathrm{m}$ (Fig. 1a) were mixed with ammonium bicarbonate (NH4) HCO3 (Fig. 1b) powders with irregular shape and a size distribution between 100 and $500 \mu \mathrm{m}$. The purpose of the latter being to act as a spacer, in order to obtain a material with large pores. The initial powders were observed with the aid of a Field Emission Scanning Electron Microscope (FESEM) Mira3 LMU, Tescan (Fig. 1), in order to determine their shape and size. The volume fraction of (NH4)HCO3 particles was 30 vol.\%. First, the mixture was prepared in a turbula for 30 min. Next, $10 \%$ by weight of PVA was added as a binder. The mixture was then poured into an $8 \mathrm{~mm}$ diameter stainless steel die and the powders were pressed at $500 \mathrm{MPa}$ by means of an Instron 1150 universal machine to obtain cylindrical compacts with a height of $12 \mathrm{~mm}$. The (NH4) HCO3 used as a pore former was removed at $180^{\circ} \mathrm{C}$ during 6 hours in an electric furnace and then, the PVA was removed by slow heating the samples at $5^{\circ} \mathrm{C} / \mathrm{min}$ up to a final temperature of $500{ }^{\circ} \mathrm{C}$ with a dwell time of 40 min under a high purity argon atmosphere. The sintering step was performed in a Linseis $\mathrm{L} 75 \mathrm{~V}$ vertical dilatometer at $1300^{\circ} \mathrm{C}$ with a sintering plateau of $1 \mathrm{~h}$ under a high purity protective atmosphere mentioned above. The density of each sintered sample was calculated from the measurement of its mass and volume. The relative density (D) is defined as the weight density of the compact divided by the theoretical density of the dense Ti6Al4V alloy, whereby, the pore volume fraction can be obtained by taking the 1-D value. The sintered samples dedicated to SEM observation were cut and the surface was metallographically prepared by grinding and polishing using abrasive $\mathrm{SiC}$ sandpaper and alumina powders. A cross-sectional image of the sintered sample is presented in Fig. 1c, where it is clearly appreciated the pores generated by the salt.

\subsection{Image acquisition and processing}

The 3D images were acquired with a Zeiss 510 Xradia Versa computerized microtomographer (CMT), before and after the compression test. 1600 projections were taken around $360^{\circ}$ of the sample with a CCD camera of $1024 \times 1024$ pixels with a voxel resolution of about $10 \mu \mathrm{m}$. A virtual $2 \mathrm{D}$ slice of the initial image is shown in Fig. 2a. In order to perform an analysis of the porosity, the whole 3D image volumes were filtered with the plug-ins included in the Image J program, both; unsharp mask and median filters were used to enhance the contrast between grains and pores, as it can be seen in Fig. 2b. Then, a separation of the solid and empty phases as a function of the gray level, which is obtained by absorption of the radiation of the sample that is determined by the atomic number of the material. In this case, the void has a small absorption compared to the Ti6Al4V. A thresholding procedure was then applied to separate the solid and the porous phases. We use a manual thresholding constrained with the global relative density that was previously measured on the whole sample from mass and volume. In the obtained binary images, the solid phase was represented by a voxel intensity of 255 and the pore phase by a voxel intensity of 0 . Then, in order to improve the quality of the binary images, successive morphological operations such as opening and closings with an octahedron as structural element were applied. Opening operation allows to eliminate small islands and sharp peaks or capes in the image. On the contrary, closing operation smooths the contours and help us to eliminate small holes and, fills gaps on the contours. The image after the morphological operations is shown in Fig. 2c. Finally, the pore volume fraction was calculated by dividing the number of voxels corresponding to the pore phase by the total number of voxels in the image. The pore size distribution was estimated by measuring the pore volume accessible to an octahedral structural element, which starts with a size of 2 voxels per side and it increases at each step, this methodology has been used by different authors to determine a distribution of pores when porosity is interconnected for both, metallic and ceramic foams ${ }^{16-18}$.
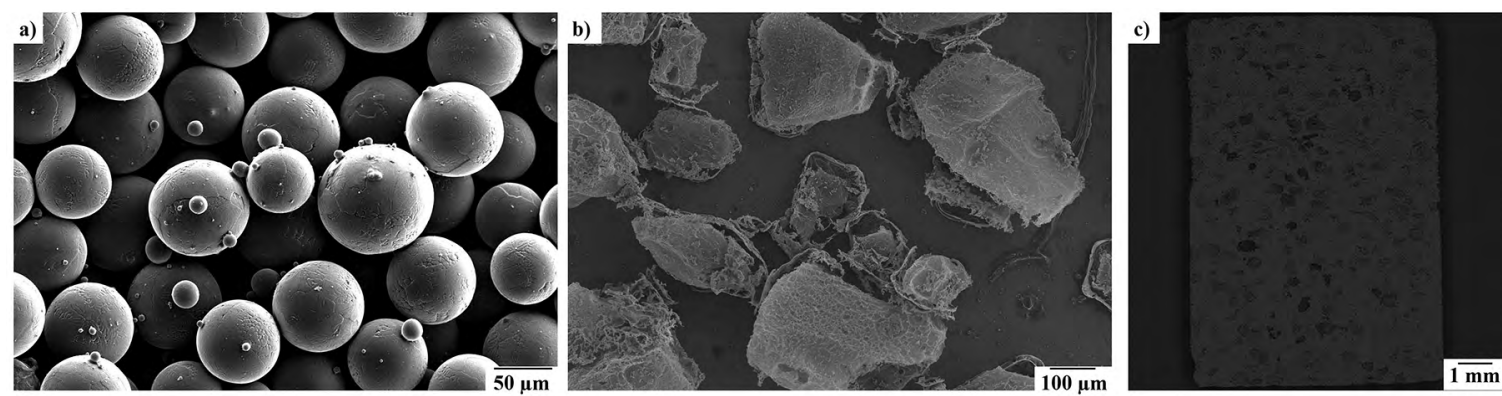

Figure 1. Initial powders, a) Ti-6Al-4V, b) ammonium bicarbonate and c) cross section of the porous sample after sintering. 


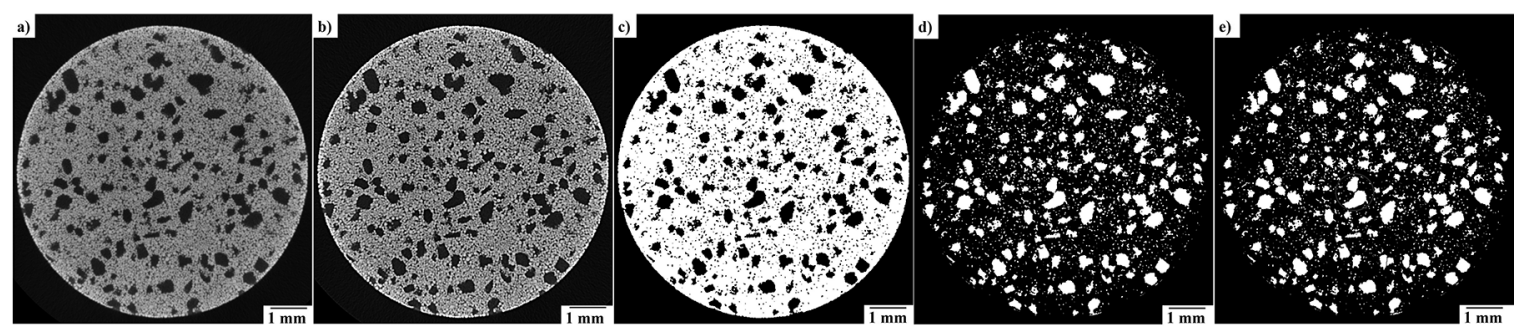

Figure 2. Virtual 2D slices of the initial image a), filtered image b), binary solid image c), binary pores image d) and binary pores segmented image e).

In order to determine the interconnectivity of pores from the binary $3 \mathrm{D}$ images, first, the porosity was set with an intensity of 255 and the solid phase at 0 , Fig. 2d. Second, we labeled these objects, and we obtained a new volume to identify every object in the image with a different grey value. Third, as the interconnected porosity was the largest pore inside the sample, we could extract it from the image by thresholding the image with the grey level value of that object that represented the pore. Finally, we calculated the volume fraction of the remaining objects in the image and, the percentage of closed porosity was obtained by the difference in the total existing porosity ${ }^{19}$.

With the aim to assess the coordination number of large pores, a segmentation procedure was achieved to separate the large pores as individual objects inside the 3D image. This was achieved using the watershed method used by different authors for segmented particles ${ }^{14,20}$ with the aid of the Avizo software. This procedure helps us to consider large pores as individual object in the 3D image, Fig. 2e. This image allows us to calculate additional information of the pore, like sphericity and coordination number.

The sphericity was defined as the sphere's surface area enclosing the same volume as the 3D object (eq. 1):

$$
S_{p}=6 V \sqrt{\frac{\pi}{S^{3}}}
$$

where $\mathrm{Sp}$ is the sphericity, $\mathrm{V}$ and $\mathrm{S}$ are the volume and the surface, respectively. This classic parameter, derived from the Saltykov 3D shape index ${ }^{21}$ was used to describe the shape of rocks ${ }^{18}$ and, it is an indicator of the irregularity of particles. As Sp approaches to a value of 1, the particle is consider to be spherical in shape.

The pore coordination number $(Z)$ was estimated as follows: contact areas were obtained from intermediate images of the particle separation process. In those images, each object (particle or contact area) was tagged and labeled, so that the contacts associated to each pore (distance $\leq 1$ voxel) were identified, as well as list of pores touching it. The mean coordination number was calculated for the whole volume. This method induces a slight overestimation of the coordination number due to the nonzero image resolution ${ }^{22}$, which can be corrected as a function of the resolution and the average pore size as proposed by Vagnon et al. ${ }^{20}$.

\subsection{Compression tests}

After sintering, the lower and upper surfaces of the sample were polished prior to the compression tests performed under ASTM D695-02 using a universal mechanical testing machine; Instron 1150. Tests were performed with a Strain rate of $0.5 \mathrm{~mm} \cdot \mathrm{min}^{-123}$. Young's modulus (E) and yield stress $\left(\sigma_{\text {yield }}\right)$ were calculated from the stress-strain curve.

\section{Results and discussion}

3D images of the whole sample after sintering are shown in Fig. 3. The distribution of large pores created with the salt particles inside the sample seems to be good since they are randomly distributed in the whole volume without large agglomerates, as it can be noticed in Fig. 3a. A 3D rendering of the sintered Ti6Al4V particles is shown in Fig. 3b. It is clearly noticed the large pores as well as the roughness on the surface, which is mainly due to interconnected large pores. Fig. $3 \mathrm{c}$ on the other hand, shows the porosity in 3D inside the sample. It is observed that pores have a rounded shape as a result of introduction of salt particles. From these images, it was found that the elimination of the pore-former particles did not generate cracks or defects in addition to the porosity. Fig. $3 \mathrm{~d}$ shows the pores segmented as individual objects inside the 3D image, where each color represents a different large pore.

The stress-strain curve of the compression tests performed on the porous samples is presented in Fig. 4. Initially, tests were carried out until complete failure and the deformation value was observed to be very high, around $50 \%$ for samples with $45 \%$ porosity. The value of the yield stress was 80 $\mathrm{MPa}$ and the value of the Elastic Modulus was found to be $8 \mathrm{GPa}$. In this figure, another compression test is also depicted, which was interrupted at a deformation of $10 \%$. This sample was observed before and after the compression test by X-ray microtomography in order to follow the changes in the porosity during testing. As it can be seen, the reproducibility of the test is very good, which is due to the fact that the volume fraction of pores obtained for samples varied only around $2 \%$. The Elastic Modulus values reported for different types of human bones range from 1 to $20 \mathrm{GPa}$ and yield values between 4 and $200 \mathrm{MPa}$, depending on the type of bone; spongy or cortical ${ }^{11,24-26}$. The values obtained in this study, are in the range of applications for implants. 

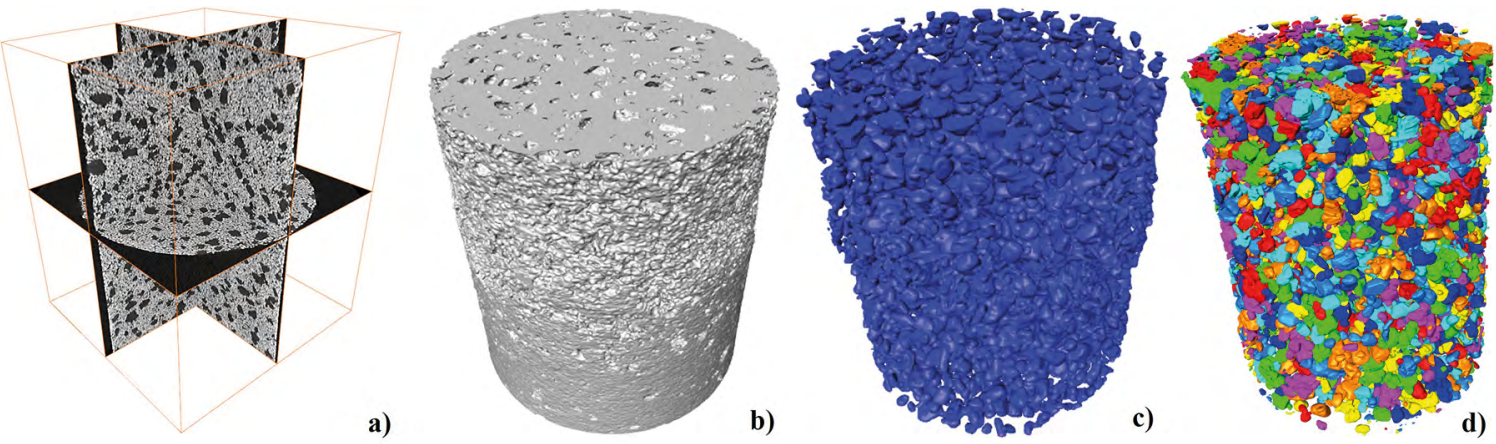

Figure 3. 3D images of porous sample after sintering a) multiortoslices, b) 3D representation of solid phase, c) pores inside of the sample and d) pores segmented.

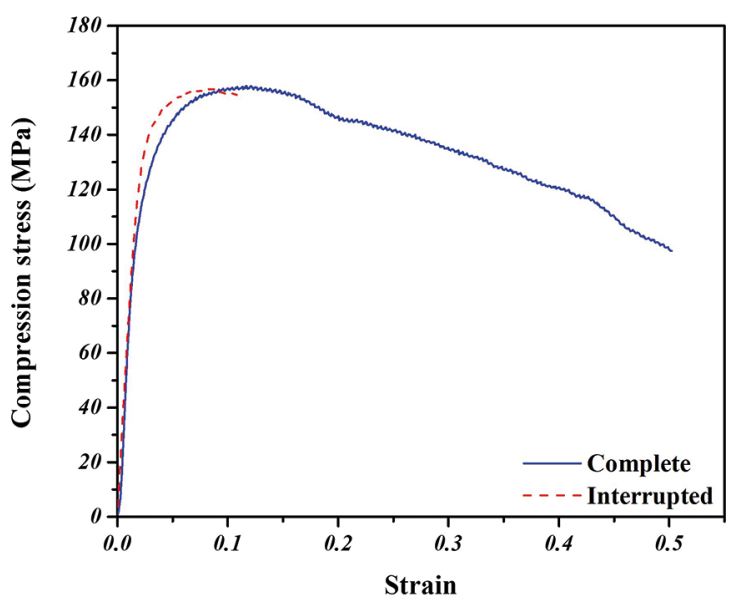

Figure 4. Stress-strain curve of porous samples.

Fig. 5a shows a 2D virtual slice of the same cross section of the above mentioned sample before compression, while Fig. $5 \mathrm{~b}$ shows the virtual slice after the interrupted compression test. As it can be seen, there is a preferential deformation zone at the top of Fig. 5b, where the initial porosity decreased drastically due to the plastic deformation suffered where $10 \%$ of plastic deformation was achieved. In the lower part of the image, the initial pores can be distinguished, although, modifications in shape and size are seen but, with the same distribution within the sample. This anisotropic behavior in the deformation indicates that the induced porosity generates certain instability in the sample leading to the weaker walls of the structures to collapse, while the porosity is being closed progressively supporting more deformation. The contribution of porosity to the ductility of the material was previously reported by Biswas and co-workers ${ }^{27}$.

A 3D representation of the volume affected by compression test is shown in Fig. 6. The generation of cracks in the face perpendicular to the load is also noticed, Fig. 6a. It can be seen that cracks grow through large pores that are also deformed, whereas their volume is reduced, Fig. 5b. The pore shape shown in Fig. 3c is no longer distinguishable in Fig. 5b

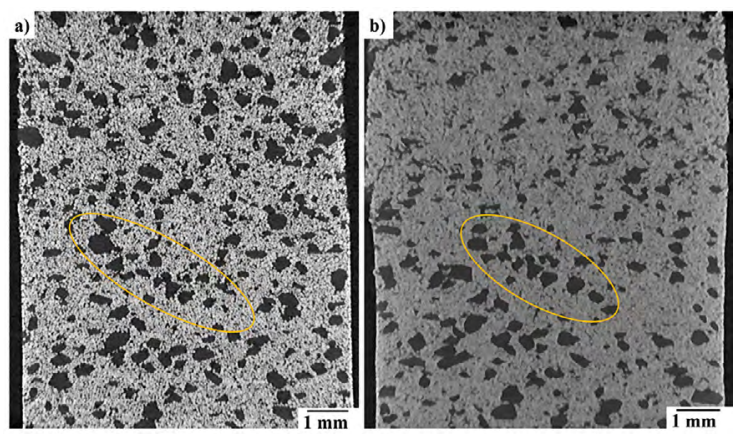

Figure 5. 2D transverse slices of porous samples, (a) before and (b) after interrupted compression test.

because of the deformation of large pores. Some elongated pores in the perpendicular direction to the compression load are observed, and most of the porosity became a complex shape that is still interconnected.
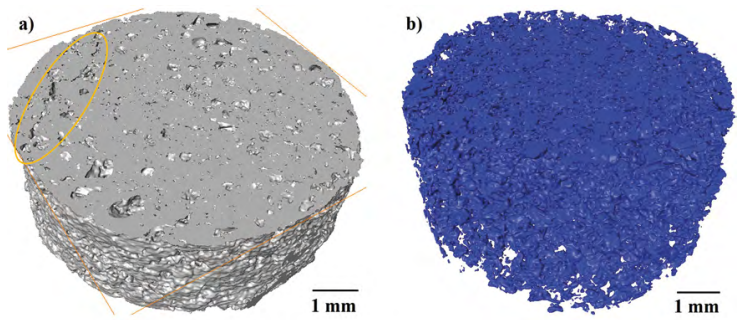

Figure 6. 3D representation of the volume affected by the compression (a) in the solid phase and (b) porosity.

The pore size distribution of the samples before and after the compression tests is presented in Fig. 7. It was found that in the sample before compression, $30 \%$ of the total pores are less than $50 \mu \mathrm{m}$, which would indicate that the remaining porosity concentrates between particles. The remaining porosity remains because the initial Ti6Al4V particles are coarse, thus, the sintering is slow and the densification is gradually reduced during the process. On the other hand, it can be seen that $70 \%$ of the remaining pores correspond to 
pores larger than $100 \mu \mathrm{m}$, which are related to the size of the ammonium bicarbonate particles introduced to generate the voids. This pore size distribution indicates that there is almost no agglomeration of salt particles, since the pores would be larger than $500 \mu \mathrm{m}$. After compression, it is possible to observe that the volume fraction of pores smaller than $50 \mu \mathrm{m}$ is reduced up to the half of its initial value. This indicates that the deformation found in the compression curves generates a rearrangement of the particles by closing the smaller pores first and, in turn, creating some larger and longer pores by the coalescence of these. It is also possible to note that the pore fraction with pores larger than $200 \mu \mathrm{m}$ is slightly increased, however, a reduction in the global porosity was the result of the deformation during compression as it can be seen in Table 1 .

With the aim of understanding the evolution of the porosity. Fig. 8 shows 3D images of the pores in the collapsed and regular deformation regions (as indicated in Fig. 5b), before and after the compression test. First, for the collapsed region, it can be observed that most of the small pores existing

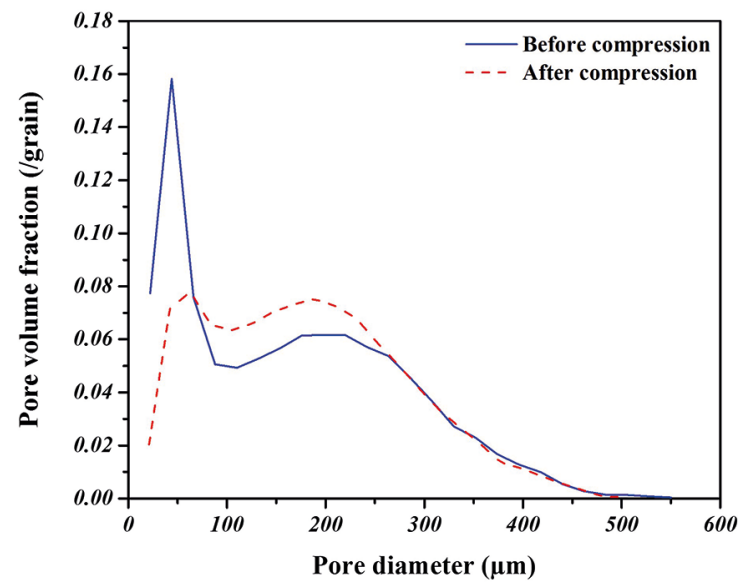

Figure 7. Pore size distribution before and after compression test. before compression are considerably reduced, see Figs. $8 \mathrm{a}$ and $8 \mathrm{~b}$. This observation corroborates the reduction of pores smaller than $50 \mu \mathrm{m}$, as it was quantified in Fig. 7. The interconnected large pores are forming new and elongated pores due to the deformation suffered which in turn, leads to fissures. It can be intuited that the porosity favors the path of the cracks created during the compression test. On the other hand, pores located in the regular deformation zone, Figs. $8 \mathrm{c}$ and $8 \mathrm{~d}$ did not show a crack-like formation but, it is noticed that smaller pores were closed, and the larger ones become rounded. It was also found that interconnectivity between large pores before compression is increased by the interparticle pores that disappears during compression and the final interconnectivity after compression is mainly due to contact between large pores. This observation explains why the pore interconnectivity is reduced and the pore coordination number increased, as shown in Table 1.

Quantitative data of porosity before and after compression are presented in Table 1. For this case, the entire porosity of the sample was taken into account, thus, the average values do not undergo such drastic changes since the collapsed region is a third of the whole sample. It was found that the volume fraction of the porosity is reduced up to $11 \%$, corresponding to the value of the macroscopic deformation undergone during the compression test. The interconnectivity of the porosity was also reduced by $10 \%$. This is mainly due to the elimination of interparticle pores; either by rearrangement of particles and/or by coalescence with larger pores resulting in fissures. This is in agreement with the increment of $7 \%$ in the median pore size, which might be due to the coalescence with smaller pores and the elongation of the pores due to the deformation. Finally, the increase in the coordination number of large pores indicates that the cracks are formed by the connection between the large pores, since it increases by $35 \%$, although, the total value of interconnectivity of the porosity was reduced.

Table 1. Characteristics of the porosity evaluated from 3D images.

\begin{tabular}{lccccc}
\hline Sample & $\begin{array}{c}\text { Volume fraction } \\
(\%)\end{array}$ & $\begin{array}{c}\text { Interconnectivity } \\
(\%)\end{array}$ & $\begin{array}{c}\text { Median size pore } \\
(\mathrm{d} 50)\end{array}$ & Sphericity & $\begin{array}{c}\text { Coordination } \\
\text { number }\end{array}$ \\
\hline Before compression & 39.4 & 99.5 & 228 & 0.662 & 2.01 \\
After compression & 34.7 & 90 & 245 & 0.726 & 2.72 \\
\hline
\end{tabular}
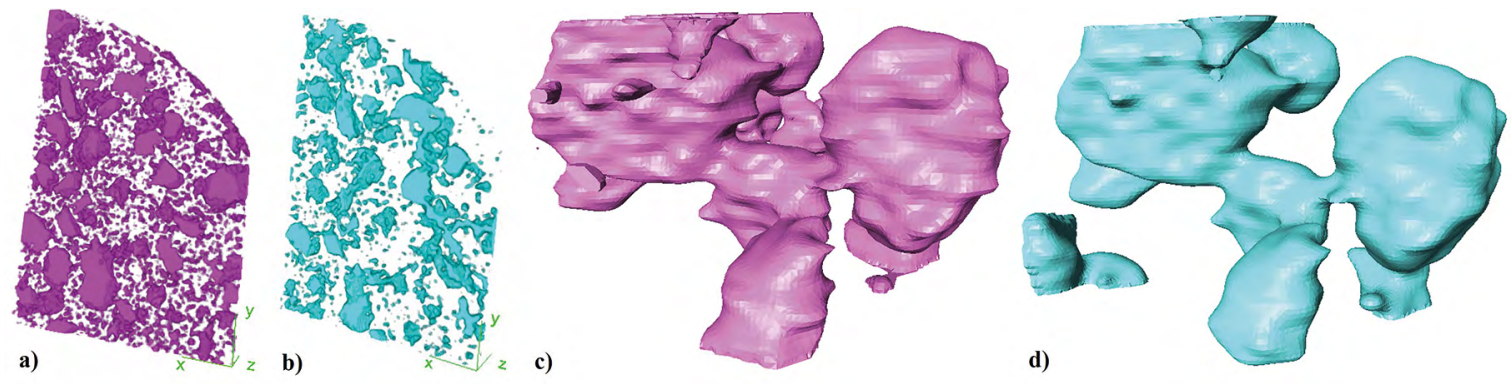

Figure 8. 3D images of porosity before and after compression test in two different zones, a) and b) failure volume; c) and d) deformed zone. 


\section{Conclusions}

The compression behavior of porous samples of the Ti6Al4V alloy for potential surgical implant applications was studied in which large pores were artificially created and characterized by X-ray microtomography. It was determined that the porosity decreases the mechanical properties of the alloy up to values that are in the range of those reported for human bones. It was also found that the porosity is completely interconnected, which would favor the passage of body fluids between the implant and the bones of patients.

It was observed that the fracture of the samples is perpendicular to the load, contrary to cast materials which usually fail at $45^{\circ}$ angle, which means that failure of porous samples occurs through layers from the top to the bottom of the sample. This behavior is clearly induced by the size, shape and orientation of large pores artificially introduced in the matrix of Ti6Al4V particles. Thus, the cracks are generated and grow through the large pores breaking the interparticular necks formed during sintering.

Finally, it was concluded that the methodology used for the creation of large pores is adequate, since the distribution of the large pores inside the sample is well developed and the results of stress and Elastic Modulus are replicable.

\section{Acknowledgement}

The authors would like to thank to CIC of the UMSNH and ECOS M15P01 for the financial support and the facilities to develop this study. The Laboratory "Lumir" Geosciences of the UNAM, Juriquilla for the 3D image acquisition and processing al also acknowledge with thanks.

\section{References}

1. Dewidar MM, Khalil KA, Lim JK. Processing and mechanical properties of porous $316 \mathrm{~L}$ stainless steel for biomedical applications. Transactions of Nonferrous Metals Society of China. 2007;17(3):468-473.

2. Bender S, Chalivendra V, Rahbar N, El Wakil S. Mechanical characterization and modeling of graded porous stainless steel specimens for possible bone implant applications. International Journal of Engineering Science. 2012;53:67-73.

3. Eriksson M, Andersson M, Adolfsson E, Carlström E. Titaniumhydroxyapatite composite biomaterial for dental implants. Powder Metallurgy. 2006;49(1):70-77.

4. Rack HJ, Qazi JI. Titanium alloys for biomedical applications. Materials Science and Engineering: C. 2006;26(8):1269-1277.

5. Oksiuta Z, Dabrowski JR, Olszyna A. Co-Cr-Mo-based composite reinforced with bioactive glass. Journal of Materials Processing Technology. 2009;209(2):978-985.

6. Dourandish M, Godlinski D, Simchi A, Firouzdor V. Sintering of biocompatible P/M Co-Cr-Mo alloy (F-75) for fabrication of porosity-graded composite structures. Materials Science and Engineering: A. 2008;472(1):338-346.
7. Bandyopadhyay A, Espana F, Balla V, Bose S. Influence of porosity on mechanical properties and in vivo response of Ti6Al4V implants. Acta Biomaterialia. 2010;6(4):1640-1648.

8. Oshida Y. Bioscience and bioengineering of titanium materials. Amsterdam: Elsevier; 2010.

9. Carvalho DRD, Carvalho PSPD, Magro Filho O, Mello JDBD, Beloti MM, Rosa AL. Characterization and in vitro cytocompatibility of an acid-etched titanium surface. Brazilian Dental Journal. 2010;21(1):3-11.

10. Geetha M, Singh AK, Asokamani R, Gogia AK. Ti based biomaterials, the ultimate choice for orthopaedic implants - A review. Progress in Materials Science. 2009;54(3):397-425.

11. Ryan G, Pandit A, Apatsidis DP. Fabrication methods of porous metals for use in orthopaedic applications. Biomaterials. 2006;27(13):2651-2670.

12. Camron HU, Pilliar RM, Macnab I. The rate of bone ingrowth into porous metal. Journal of Biomedical Materials Research Part A. 1976;10(2):295-302.

13. Bobyn JD, Pilliar RM, Cameron HU, Weatherly GC. The optimum pore size for the fixation of porous-surfaced metal implants by the ingrowth of bone. Clinical Orthopaedics and Related Research. 1980;150:263-270.

14. Olmos L, Takahashi T, Bouvard D, Martin CL, Salvo L, Bellet D, Di Michiel M. Analysing the sintering of heterogeneous powder structures by in situ microtomography. Philosophical Magazine. 2009;89(32):2949-2965.

15. Moreno-Atanasio R, Williams RA, Jia X. Combining X-ray microtomography with computer simulation for analysis of granular and porous materials. Particuology. 2010;8(2):81-99.

16. Olmos L, Martin CL, Bouvard D, Bellet D, Di Michiel M. Investigation of the sintering of heterogeneous powder systems by synchrotron microtomography and discrete element simulation. Journal of the American Ceramic Society. 2009;92(7):14921499.

17. Appoloni CR, Fernandes CP, Innocentini MDDM, Macedo Á. Ceramic foams porous microstructure characterization by X-ray microtomography. Materials Research. 2004;7(4):557-564.

18. Lin CL, Miller JD. 3D characterization and analysis of particle shape using X-ray microtomography (XMT). Powder Technology. 2005;154(1):61-69.

19. Olmos L, Bouvard D, Salvo L, Bellet D, Di Michiel M. Characterization of the swelling during sintering of uniaxially pressed copper powders by in situ X-ray microtomography. Journal of Materials Science. 2014;49(12):4225-4235.

20. Vagnon A, Rivière JP, Missiaen JM, Bellet D, Di Michiel M, Josserond C, Bouvard D. 3D statistical analysis of a copper powder sintering observed in situ by synchrotron microtomography. Acta Materialia. 2008;56(5):1084-1093.

21. Saltykov SA. Stereometrische Metallographie. Leipzig: VEB Deutscher Verlag für Grundstoffindustrie; 1974.

22. Marmottant A, Salvo L, Martin CL, Mortensen A. Coordination measurements in compacted $\mathrm{NaCl}$ irregular powders using $\mathrm{X}$-ray microtomography. Journal of the European Ceramic Society. 2008;28(13):2441-2449. 
23. Cheng XY, Li SJ, Murr LE, Zhang ZB, Hao YL, Yang R, Medina F, Wicker RB. Compression deformation behavior of Ti-6Al$4 \mathrm{~V}$ alloy with cellular structures fabricated by electron beam melting. Journal of the Mechanical Behavior of Biomedical Materials. 2012;16:153-162.

24. Mish CE, Qu Z, Bidez MW. Mechanical properties of trabecular bone in the human mandible: implications for dental implants treatment planning and surgical placement. Journal of Oral and Maxillofacial Surgery. 1999;57(6):700-6.
25. Reilly DT, Burstein AH. The elastic and ultimate properties of compact bone tissue. Journal of Biomechanics. 1975;8(6):393IN9397396IN11405.

26. Xie F, He X, Cao S, Mei M, Qu X. Influence of pore characteristics on microstructure, mechanical properties and corrosion resistance of selective laser sintered porous Ti-Mo alloys for biomedical applications. Electrochimica Acta. 2013;105:121-129.

27. Biswas N, Ding J, Balla V. Deformation and fracture behavior of laser processed dense and porous Ti6Al4V alloy under static and dynamic loading. Materials Science and Engineering A. 2012;549:213-221. 